\title{
Impact of Coordination Mechanisms on the Collaborative Planning Process Components
}

\author{
M.M.E. Alemany, F. Alarcón, and A. Ortiz \\ Research Centre on Production Management and Engineering (CIGIP), Universidad \\ Politécnica de Valencia, Camino de Vera s/n, Valencia 46022, Spain \\ mareva@omp.upv.es, faualva@omp.upv.es, aortiz@cigip.upv.es
}

\begin{abstract}
In this paper a conceptual framework for characterizing the Supply Chain (SC) Collaborative Planning (CP) under a process perspective is presented. When analyzing the $\mathrm{CP}$ process view, the definition of coordination mechanisms among SC members results essential. With the aim of identifying the possible coordination mechanisms that SC members can implement, the structural elements necessary to define them are proposed. Finally, the impact that the coordination mechanisms structural elements have on the definition of the $\mathrm{CP}$ process is presented. The knowledge of the $\mathrm{CP}$ process, the coordination mechanisms and their relationship constitutes the basis for the latter identification of potential activities that can be supported by decision tools and for the partially or totally automation of the process.
\end{abstract}

Keywords: Collaborative Planning Process, Supply Chain, Coordination Mechanisms.

\section{Introduction}

Collaborative Planning (CP) can be defined as a joint decision making process for aligning plans of individual Supply Chains (SC) members with the aim of achieving a certain degree of coordination [1]. Because CP belongs to the SC coordination problems category, CP decisions related to the mid and short-term temporal levels must be integrated (temporal integration). Furthermore, for each temporal level, the CP decision-making can be centralized if only one decision-maker exists or distributed, in case several decision makers exist. The plans of these different decision-makers must be also integrated (spatial integration). Indeed, [2] identifies as a major CP challenge to simultaneously achieve both types of integration. In a collaborative distributed context different decision-makers should share some information and should make several decision cycles involving repetitive sequence of decisions until a stopping criteria is achieved, in case negotiation exists. In this situation, the knowledge about the process results essential. Indeed, different authors strength the importance of analyzing the collaboration under a process perspective.

[3] define collaboration as a process in which entities share information, resources and responsibilities to jointly plan, implement and evaluate a program of activities to achieve a common goal and therefore jointly generating value. From the process perspective, there are different works that report flowcharts representing the $\mathrm{CP}$ process 
for a specific distributed decision environment (e.g. [4], [5], [6]). However, these CP processes are specifically designed for particular situations being necessary a generic approach that help in the definition, characterization and analysis of CP processes in general. In this sense, [7] conclude that a process will be defined when the answers to the following questions are known: (a) What activities are to be carried out?, (b) Who is responsible to carry them out and with what?, (c) When and how are they to be carried out?, (d) The process inputs, (e) The process outputs, (f) The process objectives, and (g) The performance indicators.

Through the $\mathrm{CP}$ process approach it is possible to implement the coordination mechanisms that link the SC members' plans. In this sense, [8] consider that the coordination mechanisms states how the SC members' relationship is operationalized (e.g. rules and protocols to exchange information, which decisions must be taken, by whom and when). [9] affirms that the coordination mechanisms for Distributed Decision-Making systems for a two-level hierarchical environment are: anticipation, instruction and reaction. [10] categorize the supply network (SN) coordination mechanisms into four classes: SN contracts, Information Technology, Information Sharing and Joint Decision Making. [11] establish that a coordination mechanism for a decentralized SN system should include at least three components: (i) an operational plan to coordinate the decisions and activities of SN members, (ii) a structure to share information among the members, (iii) an incentive scheme to allocate the benefits of coordination so as to entice the cooperation of all members. [1] states that the interaction between the parties involved in a $\mathrm{CP}$ scheme can be documented by a protocol defined by the following structural elements: the incorporation or not of a mediator, the initial solution, the number of rounds and the number of offers to be exchanged (stopping criteria) and the final results $\mathrm{SN}$ members can expect. In the context of agent technology applied to $\mathrm{CP},[12]$ present the main characteristic of automated negotiations (collaboration level, number of participants, number of issues, decision sequence and learning ability), decision mechanisms that can be followed by agents (game theoretic negotiation, argumentation-based negotiation, auctions and heuristic based negotiation). [13] proposes an approach that breaks from the hypothesis that planning must always be conducted in the same way. By using multi-behaviour agents they propose to provide planning agents with the ability to adapt their planning behaviours according to changes in the environment.

From the literature reviewed, it can be concluded that there is a strong relationship between the $\mathrm{CP}$ process view and the coordination mechanisms that, in our opinion, requires a deeper analysis. This paper attempts to contribute to clarify this relationship being, therefore, the objective of this paper manifold: 1) to propose a conceptual framework for the $\mathrm{CP}$ process that support the characterization, definition and analysis of the $\mathrm{CP}$ under a process perspective, 2) to identify the structural elements of the $\mathrm{CP}$ coordination mechanisms and 3 ) to define the impact of the coordination mechanisms structural elements on the components of the CP process. The knowledge of the $\mathrm{CP}$ process, the coordination mechanisms and their relationship is essential for the latter development of any supporting decision-making tool and the partially or totally automation of the process.

The rest of the paper is organized as follows. In section 2 the conceptual framework for characterizing the $\mathrm{CP}$ process is described. The coordination mechanisms structural elements for the $\mathrm{CP}$ are defined in section 3 and their impact on the $\mathrm{CP}$ process components is shown in section 4 . Finally, section 5 reports some conclusions. 

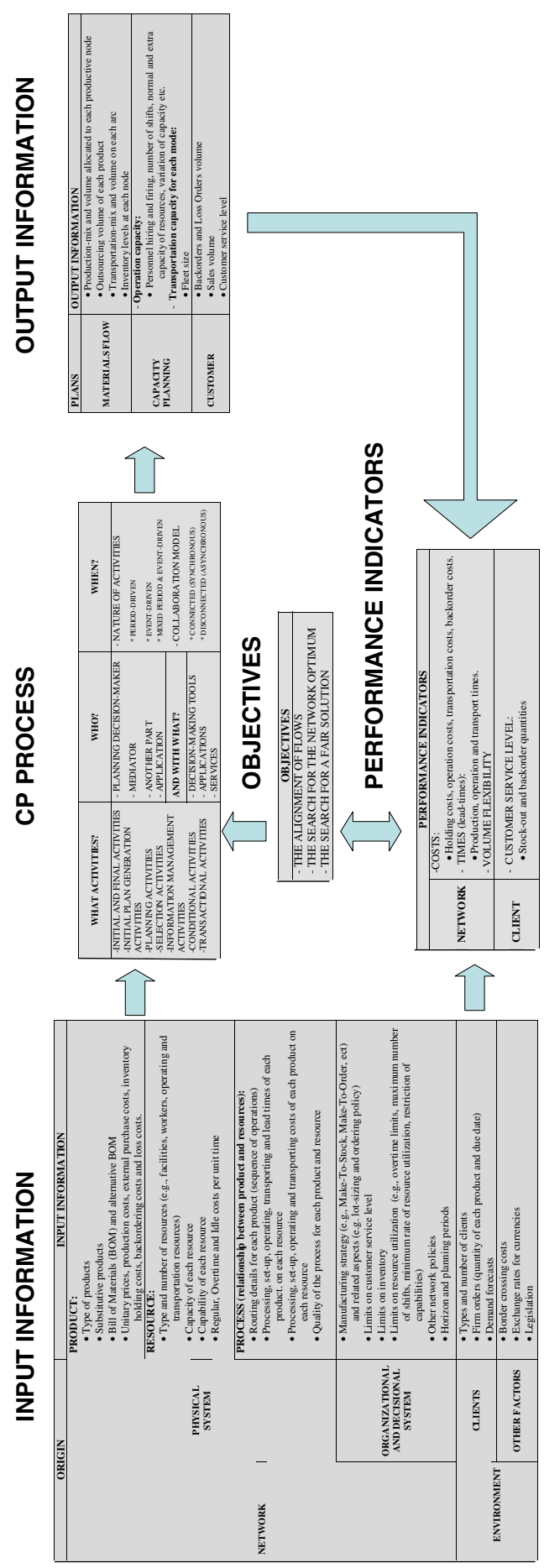

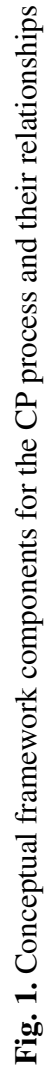




\section{Conceptual Framework for the CP Process Characterization}

As [7], we consider that a process is characterized when the answers to the questions (a)-(g) are known (Table 1). The answer to each question corresponds with the characterization of a specific component of the $\mathrm{CP}$ process conceptual framework. For a deeper explanation of the $\mathrm{CP}$ process conceptual framework, the reader is referred to [14].

The order to answer each question is not sequential, i.e., during the $\mathrm{CP}$ process characterization the answer to some questions are interrelated. Furthermore, though global process inputs, outputs, objectives and indicators exist and should be determined for the entire CP process, each activity of the process presents its own inputs, outputs, objectives and performance indicators.

Therefore, to properly define the coordination mechanisms in SCs, the exchanged information among activities should be reflected. This internal information flow is missing if the only analysis of inputs and outputs under a general process perspective as a whole is performed.

As a consequence, the questions (a)-(g) will be answered not only from a whole process perspective but also, when it will be necessary, from an activity perspective. In Fig. 1, a more detailed view of each conceptual framework component and the relationship between them are provided.

Table 1. Components of the CP process conceptual framework

\begin{tabular}{|l|l|l|}
\hline \multicolumn{1}{|c|}{ THE PROCESS ITSELF } & \multicolumn{1}{|c|}{ THE INFORMATION } & \multicolumn{1}{c|}{ EVALUATION ASPECTS } \\
\hline $\begin{array}{l}\text { (a) What activities are to be carried out? } \\
\text { (b) Who is responsible, who has the authority } \\
\text { to carry them out and with what? }\end{array}$ & (d) The process inputs, & (f) The process objectives, \\
(c) When and how are they to be carried out? & (e) The process outputs, & (g) The performance indicators \\
\hline
\end{tabular}

\section{Structural Elements of CP Coordination Mechanisms}

In this section, the structural elements that should be specified in order to characterize coordination mechanisms in a CP context are proposed. Through this characterization, an analysis of possible alternatives for implementing the interdependence relationships between SC members will be made. The proposed structural elements are derived from the analysis of the conceptual literature cited in the introduction section combined with the abstraction and generalization of particular CP models and the own experience of the authors.

Number of decision-makers: it makes reference to the number of SC members that are either under the responsibility of a SC planning domain at a certain planning temporal level or should coordinate and integrate the different plans of other decisionmakers (a mediator). It is of relevance the distribution of the decision-makers along the SC tiers and the number of decision-makers in each one. The most common situations discussed in the literature [12] are centered on a two tier SC and are the following: one-to-one, one-to-many and many-to-many negotiations. 
Collaboration level: it represents the degree of interest in decision makers' performance. Agreed collaboration level is intensely related with the final results decisionmakers should expect, the decision-makers' goal congruence, the information the SC members are in position to share and the trust between them. It is possible distinguish between three main levels of collaboration:

- Strong collaboration (altruistic partners that puts the SC goals before its local goals)

- Low collaboration (self-interested partner that makes decisions mainly following its local goals)

- Balanced collaboration (any degree of balance between the two previous levels)

Interdependence relationships nature: the sharing of power between SC decisionmakers could be not homogeneous. The relative position of each decision-maker in the SC depends on different factors that impact the influence of each decision-maker in the definition of the interdependence relationships. Two differentiate cases could be distinguished:

- Hierarchical: the sharing of power between SC members is not homogeneous and, then, $\mathrm{CP}$ interdependence relationships are dominated by decision makers with more power in the SC. Their leadership could be reflected in the interdependence relationships in several ways (e.g., defining the negotiation stopping criteria)

- Non-hierarchical: all the SC members are recognized with the same power and, therefore, all the interdependence relationships are equally agreed.

Interdependence relationships type: because SC planning decisions are made at different temporal levels and at each temporal level different decision-makers could exist, two different types of plans integration should be distinguished:

- Temporal integration: it involves coordinating planning decisions across different timescales or across various levels of decision-making (tactical and operational). This collaboration type is always hierarchical.

- Spatial integration: it involves coordinating the plans of different decisionmakers at the same temporal level. Depending on the sharing of the power between SC members it could be hierarchical or non-hierarchical.

Number of coordination mechanisms: it refers to the number of different protocols under which the different decision-makers interact.

- Unique: there is an only one protocol that is independent on the environment and other situation characteristics.

- Various: when the environment is characterized by high levels of variability, it can be advantageous to define different coordination mechanisms to work under different scenarios. In this case, different options exists:

- Pre-defined (non-learning ability): the number of coordination mechanisms are defined in advanced. The situations under which a specific mechanism should be employed and their characteristics should be specified.

- Not pre-defined (learning ability): the decision-makers have the ability to acquire experience from previous negotiations (i.e. they are able to adapt their 
strategies with changing opponents, topics, concerns and user preferences) and to adapt to the context. They can modify their local planning behaviors and/or in concordance with the rest of the decision-makers.

Criteria to select the operation under specific coordination mechanism (available time to make decisions, source of the perturbation in the environment, etc.) in case several coordination mechanism exists.

Information exchanged: for each coordination mechanism the information exchanged between the decision-makers can make reference to SC attributes and decision-makers' outputs:

- SC attributes: they consist of known characteristics of the planning SN elements and their environment (demand forecasts, capacity of facilities, operating costs, incentives, penalizations, etc.). It is known in the literature as information sharing.

- Decision-makers' outputs: they are those decision variables/ criteria values which, in some way, are passed to other decision-makers. This output data becomes input data for others activities, more specifically they become interdependent parameters of other planning activities. It is known in the literature as joint decision-making and it is the essence of collaborative planning. Depending on the possibility of changing the final value of the outputs by the interdependent decision-makers, two outputs categories could be distinguished:

o Final Decision Variables/Criteria: their values cannot be changed under any condition during the negotiation process or, simply, because there is no negotiation.

o Non-Final Decision Variables/Criteria: their values can be modified during the negotiation process due to:

- Temporal integration: disaggregation of decisions should be made for being implemented (e.g. production volume of families should be disaggregated referred to articles)

- Spatial integration: their values are adjusted before reaching the stopping criteria of the negotiation process (e.g. ordered/supplied quantities)

Information processing: the exchanged information for each coordination mechanism could be incorporated in different ways by each decision-maker. Part of the share information can be simply evaluated by other decision-makers (i.e. an ordered pattern can be evaluated by a supplier to know the value of his performance criteria when he does not deviate from the buyer pattern). Other exchanged information can be incorporated in the planning problem of a decision-maker by affecting his decisional space (introduction of constraints) and/or his criteria (penalizations or incentives for deviating from a constraint)

Decision sequence characteristics: it allows define how the coordination mechanisms will be managed.

- Beginning of the coordination mechanism: for each coordination mechanism it is necessary to specify when beginning (period, event-driven or a mixed), and how beginning (initial solution by upstream planning, downstream planning, random, by a coordinator, etc.) 
- Sequence of decisions: the order in which the different decision-makers act and the decisions simultaneously made by different SC members should be specified.

Stopping criteria of the coordination mechanism: in case negotiation exists, the conditions for ending a coordination mechanism could be defined in terms of number of rounds, limited time and the achievement of a determined aspiration level related with the final results the SN members can expect.

\section{Impact of Coordination Mechanisms Structural Elements on the CP Process Components}

In this section, the relationship between the components characterizing the $\mathrm{CP}$ under a process perspective and the structural elements of the coordination mechanisms is presented (Table 2). A grey cell in the matrix means that the definition of the specific $\mathrm{CP}$ process component is either influenced or it is dependent on the characteristics of the coordination mechanism structural element. For instance, because the number of planning activities should be at least as the number of decision-makers, a grey cell appears in the intersection of the both items.

Table 2. Relationship between CP process components and the coordination mechanisms structural elements

\begin{tabular}{|c|c|c|c|c|c|c|}
\hline \multirow{4}{*}{$\begin{array}{c}\text { COORDINATION MECHANISMS } \\
\text { Structural elements }\end{array}$} & \multicolumn{6}{|c|}{ CONCEPTUAL FRAMEWORK FOR THE CP PROCESS } \\
\hline & \multicolumn{3}{|c|}{ PROCESS ITSELF } & \multirow{3}{*}{$\begin{array}{c}\text { INFORMATION } \\
\begin{array}{c}\text { Process Inputs \& } \\
\text { Outputs (shared } \\
\text { information) }\end{array}\end{array}$} & \multicolumn{2}{|c|}{ EVALUATION ASPECTS } \\
\hline & \multirow[b]{2}{*}{$\begin{array}{c}\text { What } \\
\text { activities? }\end{array}$} & \multirow[b]{2}{*}{$\begin{array}{c}\text { Who and with } \\
\text { what? }\end{array}$} & \multirow[b]{2}{*}{$\begin{array}{l}\text { When and } \\
\text { How? }\end{array}$} & & (Ex ante) & (Ex post) \\
\hline & & & & & Objectives & $\begin{array}{c}\text { Performance } \\
\text { Indicators }\end{array}$ \\
\hline \multicolumn{7}{|l|}{ Number of SC decision-makers } \\
\hline \multicolumn{7}{|l|}{ Colllaboration level } \\
\hline \multicolumn{7}{|l|}{ Interdependence relationship nature } \\
\hline \multicolumn{7}{|l|}{ Interdependence relationship type } \\
\hline \multicolumn{7}{|l|}{ Number of coordination mechanisms } \\
\hline \multirow{2}{*}{\multicolumn{7}{|c|}{$\begin{array}{l}\text { Criteria to select a coordination mechanism } \\
\text { Information exchanged }\end{array}$}} \\
\hline & & & & & & \\
\hline \multicolumn{7}{|l|}{ Information processing } \\
\hline \multicolumn{7}{|l|}{ Decision sequence characteristic } \\
\hline Stopping criteria & & & & & & \\
\hline
\end{tabular}

Therefore, table 2 shows the impact of the coordination mechanisms on the $\mathrm{CP}$ process facilitating its properly understanding and representation. To understand the process and the relationship between its elements is, indeed, the first step and constitutes the basis to either totally or partially automated the process (e.g., by means of agent technology) or to develop decision support tools for each SC member, (e.g., mathematical programming models).

\section{Conclusions}

In a distributed decision-making environment, the way the collaborative SC members make its decisions and coordinate them results essential to plan the SC operations in a coherent manner. For this reason, there is a wide body of research that develops decision support tools for SC partners and proposes coordination mechanisms between 
them. The process view is necessary to implement the coordination mechanisms which are the essence of CP because it allows clearly define the information flows, the sequence and timing of decisions, the number of decision cycles, the stopping criteria, etc. Proper process knowledge and characterization is the first step to identify the potential activities for which developing decision support tools and to automate the process. The automation of the process can allow simulate and evaluate different coordination mechanisms under different scenarios before implementing them.

\section{References}

1. Stadtler, H.: A framework for collaborative planning and state-of-the-art. OR Spectrum, $131(1), 5-30$ (2009)

2. Grossmann, I.: Enterprise-wide optimization: A new frontier in process systems engineering. Aiche Journal 51(7), 1846-1857 (2005)

3. Camarinha-Matos, L.M., Afsarmanesh, H., Galeano, N., Molina, A.: Collaborative networked organizations - Concepts and practice in manufacturing enterprises. Computers \& Industrial Engineering 57(1), 46-60 (2009)

4. Jung, H.S., Chen, F.F., Jeong, B.J.: Decentralized supply chain planning framework for third party logistics partnership. Computers \& Industrial Engineering 55(2), 348-364 (2008)

5. Sapena, O., Onaindia, E., Garrido, A., Arangu, M.: A distributed CSP approach for collaborative planning systems. Engineering Applications of Artificial Intelligence 21(5), 698-709 (2008)

6. Jain, V., Deshmukh, S.G.: Dynamic supply chain modeling using a new fuzzy hybrid negotiation mechanism. International Journal of Production Economics 122(1), 319-328 (2009)

7. Alarcón, F., Alemany, M.M.E., Ortiz, A.: Conceptual framework for the characterization of the order promising process in a collaborative selling network context. International Journal of Production Economics 120(1), 100-114 (2009)

8. Gaudreault, J., Frayret, J.M., Pesant, G.: Distributed search for supply chain coordination. Computers in Industry 60(6), 441-451 (2009)

9. Schneeweiss, C.: Distributed decision making in supply chain management. International Journal of Production Economics 11 84(1), 71-83 (2003)

10. Arshinder, K.A., Deshmukh, S.G.: Supply chain coordination: Perspectives, empirical studies and research directions. Int. J. Prod. Econ. 115(2), 316-335 (2008)

11. Li, X.H., Wang, Q.N.: Coordination mechanisms of supply chain systems. European Journal of Operational Research 16 179(1), 1-16 (2007)

12. Forget, P., Monteiro, T., D’Amours, S., Frayret, J.-M.: Collaborative agent-based negotiation in supply chain using multi-behaviour agents, CIRRELT-2008-54, 1-21 (2008)

13. Forget, P., D'Amours, S., Frayret, J.-M., Gaudreault, J.: Study of the performance of multi-behaviour agents for supply chain planning. Computers in Industry 60, 698-708 (2009)

14. Alemany, M.M.E., Alarcón, F., Lario, F.C., Poler, R.: Conceptual Framework for the Interoperability Requirements of Collaborative Planning Process (2010) (accepted for IESA) 